\title{
Questions with inconclusive answers
}

\section{To the Editor:}

I read with great interest the study by PrATs $e t$ al. [1] entitled "Effects of antibiotics on protected specimen brush sampling in ventilator-associated pneumonia" recently published in the European Respiratory Journal. The study demonstrated that the effect of antimicrobial treatment on the diagnostic yield of the bronchoscopically retrieved protected specimen brush (PSB) must be identified according to the microorganism involved. Haemophilus influenzae and Streptococcus pneumoniae seemed particularly vulnerable to antimicrobial treatment, whereas Staphylococcus aureus, Pseudomonas aeruginosa and Acinetobacter baumanii seemed more resistant. But what do these findings implicate in terms of the usefulness of PSB in patients with suspected ventilator-associated pneumonia (VAP)? Are we to withhold antimicrobial treatment prior to diagnostic evaluation? Should we rely more widely on PSB after having corrected the apparent biases in the literature evaluating the diagnostic yield of bronchoscopic sampling?

The issue of antimicrobial pretreatment should be honestly discussed in a realistic clinical scenario. Although it is clearly inadequate to initiate antimicrobial treatment prior to diagnostic sampling when the corresponding facilities are established, these facilities may not be available under defined circumstances (e.g. during night hours and weekends). Moreover, most regimens of antimicrobial pretreatment are present because of earlier septic episodes unrelated to suspected VAP. The indication for these antimicrobial treatment courses may be debatable in some cases but will be a matter of fact in most instances. The implications of these two types of antimicrobial treatment are completely different: although antimicrobial pretreatment initiated for suspected VAP clearly reduces the diagnostic yield, particularly in pathogens involved in early onset pneumonia, it does not when initiated for other reasons $[2,3]$. In fact, it is reasonable to assume that in patients developing VAP during antimicrobial treatment, the diagnostic yield may even be increased since underlying pathogens would not have caused pneumonia if these had been appropriately covered by the current antimicrobial regimen.

With these considerations in mind, any diagnostic sampling will be most rewarding in the presence of a high amount of resistant pathogens not covered by any given initial antimicrobial treatment policy. This conclusion is in line with the fact that an institution reporting a rate of $59 \%$ resistant microorganisms in patients with suspected VAP has continuously found the best operative figures of bronchoscopic sampling [4]. However, from these associations we should learn that bronchoscopic sampling should not be continuously implemented in the routine practice of any intensive care unit (ICU). The challenge of reducing overall resistance rates by reducing antimicrobial selection pressure cannot be resolved by withholding antimicrobial treatment and implementing invasive diagnostic measures in order to establish the presence of VAP. It should be remembered that antimicrobial pretreatment is not the only confounder when applying diagnostic techniques based on quantitative cultures $[5,6]$. Instead, the preferred strategy when facing this challenge is the definition and implementation of: 1) an appropriate policy of empiric initial antimicrobial treatment; 2) an infection control programme, including strict measures in hospital environments with excess resistance rates; and 3) a critical judgment, including clinical and microbiological data, when estimating the probability of pneumonia in individual patients. Additional clues emerge from studies evaluating strategies to reduce selection pressure by short-course antimicrobial treatment regimen in patients at low risk of mortality [7].

Therefore, in my view, reopening the debate about the usefulness of bronchoscopically sampled protected specimen brush (and bronchoalveolar lavage) in patients with suspected ventilator-associated pneumonia would mean dealing with questions that have been shown to have inconclusive answers.

\section{S. Ewig}

Medizinische Universitätsklinik und Poliklinik II Bonn, Sigmund Freud Straße 25, 53127 Bonn, Germany.

\section{References}

1. Prats E, Dorca J, Pujol M, et al. Effects of antibiotics on protected specimen brush sampling in ventilatorassociated pneumonia. Eur Respir J 2002; 19: 944-951.

2. Timsit JF, Misset B, Renaud B, Goldstein FW, Carlet J. Effect of previous antimicrobial therapy on the accuracy of the main procedures used to diagnose nosocomial pneumonia in patients who are using ventilation. Chest 1995; 108: 1036-1040.

3. Souweine B, Veber B, Bedos JP, et al. Diagnostic accuracy of protected specimen brush and bronchoalveolar lavage in nosocomial pneumonia: impact of previous antimicrobial treatments. Crit Care Med 1998; 26: 236-244.

4. Trouillet JL, Chastre J, Vuagnat A, et al. Ventilatorassociated pneumonia caused by potentially drugresistant bacteria. Am J Respir Crit Care Med 1998; 157: 531-539. 
5. Ewig S, Torres A. Flexible bronchoscopy in nosocomial pneumonia. Clin Chest Med 2001; 22: 263-279.

6. Torres A, Carlet J. Ventilator-associated pneumonia. European Task Force on ventilator-associated pneumonia. Eur Respir J 2001; 17: 1034-1045.
7. Singh N, Rogers $\mathrm{P}$, Atwood CW, Wagener $\mathrm{MM}, \mathrm{Yu}$ VL. Short-course empiric antibiotic therapy for patients with pulmonary infiltrates in the intensive care unit. A proposed solution for indiscriminate antibiotic prescription. Am J Respir Crit Care Med 2000; 162: 505-511.

\section{Regular follow-up for patients irradiated for early stage nonsmall-cell lung cancer too!}

\section{To the Editor:}

It was with interest that we read the recent article by EGERMANN et al. [1] on the outcome of regular followup in patients with nonsmall-cell lung cancer (NSCLC) treated with curative resection, including cost-effectiveness analysis. We wholeheartedly agree with the conclusions of the study, but would like to extend this observation to another subset of patients, namely those with technically operable, but medically inoperable early stage NSCLC, usually treated with radiation therapy (RT) alone [2-5]. There are a number of similarities and differences between these two patient groups.

First, contrary to surgical series, RT patients developing metachronous second primary lung cancer (mSPLC) are not treated differently from those relapsing locally from their first malignancy. Owing to pre-existing comorbidities, they are always treated the same way (with RT) [6]. While some of those patients relapsing locally may be treated palliatively $[7,8]$, a number of them may be treated curatively, particularly those with recurrences confined to the bronchial stump [7-10]. In the latter, high-dose RT alone can achieve median survival times of $\leqslant 30$ months and 5-yr survival rates of $\leqslant 30 \%[7,9,10]$.

Secondly, in contrast to surgical series where second curative resections in $\mathrm{mSPLC}$ are rare clinical events, a second RT course in mSPLC diagnosed using the same criteria by MARTINI and Melamed [11] achieved 5 -yr cause-specific and overall survival of 53 and 30\% respectively, in patients with Stage I/II NSCLC [6]. In addition, there were neither RT-related treatment deaths, contrasting (30-day) preoperative mortality of $13 \%$ in the series of EGERMANN et al. [1], nor significant (high-grade) acute or late toxicity, due to the "limited" RT treatment fields used in the patients with mSPLC [6]. RT results in this patient population are, therefore, at least comparable with those of surgical series [12-16] of second lung cancer, with a resectability rate of $\sim 50 \%$, median survival times of $1-2 \mathrm{yrs}$, and 5-yr survivals ranging from $4-32 \%$ [17].

Thirdly, while we agree with EGERMANN et al. [1] that the outcome of their patient population may have been burdened by advanced age and pre-existing comorbidities, this was even more the case for the RT-treated patients who were not surgical candidates, with the exception of a few patients who refused surgery for their initial early NSCLC [2-5]. We have used a very similar follow-up approach to that of EGERMANN et al. [1] in our patient population, and, although we did not perform cost-effectiveness analysis, we are almost certain that the same would have happened with RT.

Finally, we think that more clinical research should be performed to identify patients who may be at greater risk for developing secondary cancer or cancer that is recurring. Identification of various prognostic factors, such as clinical (patient or tumour-related), laboratory and "biological", could be included, if not before, then at least as part of a comprehensive follow-up plan, in order to direct some or all of the follow-up procedures towards the subset of patients at greatest risk for developing either metachronous second primary lung cancer or local recurrence. Although this may decrease the cost-effectiveness of any follow-up programme in this patient population, it would be instantly rewarding as it would increase the ability to diagnose such patients earlier, and, therefore, offer them more curative approaches, leading to more life-years gained, which is an ultimate goal in this disease.

\section{B. Jeremic*, J. Classen", M. Bamberg\#}

*Dept of Radiotherapy, Technical University Munich, Munich, and "University Hospital, Tuebingen, Germany.

\section{References}

1. Egermann U, Jaeggi K, Habicht JM, Perruchoud AP, Dalquen P, Soler M. Regular follow-up after curative resection of nonsmall cell lung cancer: a real benefit for patients. Eur Respir J 2002; 19: 464-468.

2. Jeremic B, Shibamoto Y, Acimovic LJ, Milisavljevic $\mathrm{S}$. Hyperfractionated radiotherapy alone for clinical stage I nonsmall cell lung cancer. Int J Radiat Oncol Biol Phys 1997; 38: 521-525.

3. Jeremic B, Shibamoto Y, Acimovic LJ, Milisavljevic S. Hyperfractionated radiotherapy for clinical Stage II nonsmall cell lung cancer. Radiother Oncol 1999; 51: 141-145.

4. Ono R, Egawa S, Suemasu K, Sakura M, Kitagawa T. Radiotherapy in inoperable stage I lung cancer. Jpn J Clin Oncol 1991; 21: 125-128.

5. Hayakawa K, Mitsuhashi N, Saito Y, et al. Limited field irradiation for medically inoperable patients with peripheral stage I non-small cell lung cancer. Lung Cancer 1999; 26: 137-142. 